Pacific Journal of Mathematics

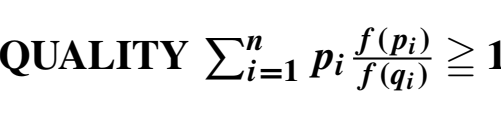




\section{ON THE INEQUALITY}

$$
\begin{gathered}
\sum_{i=1}^{n} p_{i} \frac{f\left(p_{i}\right)}{f\left(q_{i}\right)} \geqq 1 \\
\text { PAL FISCHER }
\end{gathered}
$$

We show that the inequality

$$
\sum_{i=1}^{n} p_{i} \frac{f\left(p_{i}\right)}{f\left(q_{i}\right)} \geqq 1,
$$

for all $P, Q \in A_{n}=\left\{P \in R^{n}: P=\left(p_{1}, p_{2}, \cdots, p_{n}\right) \quad\right.$ where $\sum_{i=1}^{n} p_{i}=1$ and $p_{i}>0$ for $\left.i=1,2, \cdots, n\right\}$ and some integer $n \geqq 3$, implies that $f(p)=A p^{c}$ where $A$ is an arbitrary nonzero constant and either $c \leqq-1$ or $c \geqq 0$. The converse holds as well, so that this result yields a characterization of the information gain.

1. One of the most important notions in information theory is the gain of information. It is defined by the formula

$$
I_{1}(P / / Q)=\sum_{i=1}^{n} p_{i} \log _{2} \frac{p_{i}}{q_{i}}
$$

where $P \in A_{n}$ and $Q \in A_{n}$ are complete probability distributions. The quantity $I_{1}(P / / Q)$ measures the gain of information achieved by the replacement of the a priori distribution $Q$ by the a posteriori distribution $P$. It is known that $I_{1}(P / / Q) \geqq 0$ and $I_{1}(P / / Q)=0$ iff $P=Q$.

A. Rényi introduced in [9] the measure of order $c+1$ of the gain of information by the formula

$$
I_{c+1}(P / / Q)=\frac{1}{c} \log _{2} \sum_{i=1}^{n} \frac{p_{i}^{c+1}}{q_{i}^{c}} \quad(c \neq 0) .
$$

It is easy to see that $\lim _{c \rightarrow 0} I_{c+1}=I_{1}$. The quantity (2) is only appropriate if it is nonnegative. This is the case if $c \geqq-1$, since

(i) $-1 \leqq c \leqq 0$ implies that $\Sigma p_{i}^{c+1} / q_{i}^{c} \leqq 1$, and

(ii) $c \geqq 0$ implies that $\Sigma p_{i}^{c+1} / q_{i}^{c} \geqq 1$.

On the other hand, $I_{c+1} \leqq 0$ when $c<-1$.

These facts make it desirable to characterize the functions $f$ satisfying the inequality

$$
\sum_{i=1}^{n} p_{i} \frac{f\left(p_{i}\right)}{f\left(q_{i}\right)} \leqq 1
$$


for all $P, Q \in A_{n}$, where $n$ is a fixed integer, $n \geqq 2$ and $f:(0,1) \rightarrow R-\{0\}$. Similarly, we wish to characterize those functions $f$ satisfying the inequality

$$
\sum_{i=1}^{n} p_{i} \frac{f\left(p_{i}\right)}{f\left(q_{i}\right)} \geqq 1
$$

for all $P, Q \in A_{n}$, where $n$ is a fixed integer, $n \geqq 2$ and $f:(0,1) \rightarrow R-\{0\}$.

Related questions concerning (1) were settled in [3] and these results were generalized in [5], [6] and [7]. The inequality (3), which was introduced by A. Rényi in [8], was also treated in [4]. The inequality (4) is the subject of the present paper.

Although (3) and (4) are similar in form we need an entirely different approach to solve (4). The main tool used to attack the inequality (4) is the theory of internal functions [1], [2].

2. For $n=2$, the inequality (4) has the following form

$$
p \frac{f(p)}{f(q)}+(1-p) \frac{f(1-p)}{f(1-q)} \geqq 1
$$

for all $0<p<1$ and for all $0<q<1$. The inequality (5) can be written in the form:

$$
p \frac{f(p)-f(q)}{f(q)} \geqq(1-p) \frac{f(1-q)-f(1-p)}{f(1-q)}
$$

for all $0<p<1$ and for all $0<q<1$.

In the following, we shall assume that $f>0$. From the inequality (6) we see that $f(1-q) \geqq f(1-p)$ implies $f(p) \geqq f(q)$.

If we change the roles of $p$ and $q$ in the inequality (6), then from the new inequality thus obtained and the inequality (6) we get that $f$ satisfies the inequality

$$
\begin{aligned}
\frac{q}{1-q} \frac{f(1-p)}{f(p)}[f(p)-f(q)] & \leqq f(1-q)-f(1-p) \\
& \leqq \frac{p}{1-p} \frac{f(1-q)}{f(q)}[f(p)-f(q)]
\end{aligned}
$$

for all $0<p<1$ and for all $0<q<1$. Putting $p=\frac{1}{2}$ into (5), we obtain

$$
\frac{1}{f(q)}+\frac{1}{f(1-q)} \geqq \frac{2}{f\left(\frac{1}{2}\right)}
$$


for all $0<q<1$. Inequality (8) implies that

$$
\min [f(q), f(1-q)] \leqq f\left(\frac{1}{2}\right)
$$

for all $0<q<1$, and if for some $q \min [f(q), f(1-q)]=f\left(\frac{1}{2}\right)$, then

$$
f(q)=f(1-q)
$$

Putting $q=\frac{1}{2}$ into (5), we obtain

$$
p f(p)+(1-p) f(1-p) \geqq f\left(\frac{1}{2}\right)
$$

for all $0<p<1$, i.e.

$$
\max [f(p), f(1-p)] \geqq f\left(\frac{1}{2}\right)
$$

and if $\max [f(p), f(1-p)]=f\left(\frac{1}{2}\right)$ for some $p$, then

$$
f(p)=f(1-p) .
$$

That is, we have shown that

$$
\min [f(p), f(1-p)] \leqq f\left(\frac{1}{2}\right) \leqq \max [f(p), f(1-p)]
$$

for all $0<p<1$, with equality iff $f(p)=f(1-p)$.

We have shown a slightly more general result.

Lemma 1. If $f:(0, a) \rightarrow(0, \infty)$ satisfies the inequality

$$
p \frac{f(p)}{f(q)}+(a-p) \frac{f(a-p)}{f(a-q)} \geqq a
$$

for all $0<p<a$ and for all $0<q<a$, then

$$
\min [f(p), f(a-p)] \leqq f\left(\frac{a}{2}\right) \leqq \max [f(p), f(a-p)]
$$

for all $0<p<a$, with equality iff $f(p)=f(a-p)$.

We shall need the following lemma.

LEMmA 2. If $f:(0,1) \rightarrow(0, \infty)$ satisfies the inequality (4) for a fixed integer $n \geqq 3$, then $f$ satisfies the inequality (13) for all $a \in(0,1)$. 
Proof. Let $a$ be a fixed number such that $0<a<1$. Putting $p_{1}=p, q_{1}=q, p_{2}=a-p, q_{2}=a-q$,

$$
p_{3}=q_{3}=\cdots=p_{n}=q_{n}=\frac{1-a}{n-2}
$$

into (4), we obtain that $f$ satisfies the inequality (13) for this fixed $a$.

3. Now we shall prove the following theorem:

THEOREM 1. If $f:(0,1) \rightarrow(0, \infty)$ satisfies the inequality (4) for a fixed integer $n \geqq 3$ and if $f$ is monotonic, then $f$ is differentiable.

Proof. First, we shall prove that $f$ is continuous. According to Lemma $2 f$ satisfies (13) for all $a \in(0,1)$. Using (13) and (13) with $p$ and $q$ interchanged we obtain that $f$ satisfies for all $0<a<1$ the inequality

$$
\begin{aligned}
\frac{q}{a-q} \frac{f(a-p)}{f(p)}[f(p)-f(q)] & \leqq f(a-q)-f(a-p) \\
& \leqq \frac{p}{a-p} \frac{f(a-q)}{f(q)}[f(p)-f(q)]
\end{aligned}
$$

for all $0<p<a$ and for all $0<q<a$.

From the inequality (15), which is similar to (7), we see that the continuity of $f$ at $p$ implies its continuity at $a-p$, because

$$
\lim _{q \rightarrow p} \frac{f(a-q)}{f(q)}[f(p)-f(q)]=0
$$

since $f$ is monotonic. Assume that $f$ is not continuous at a point $p_{0}$, $0<p_{0}<1$. Choosing $p_{0}<a<1$, we see that $f$ is not continuous at the point $a-p_{0}$. This is impossible because the set of points of the form $a-p_{0}$ forms an interval and since $f$ is monotonic it is continuous everywhere but on a countable set.

Using the fact that $f$ is continuous we can deduce from the inequality (15) that the differentiability of $f$ at $p$ implies its differentiability at $a-p$. Moreover, the relation

$$
\frac{p}{a-p} \frac{f(a-p)}{f(p)} f^{\prime}(p)=f^{\prime}(a-p)
$$

holds. Assume that $f$ is not differentiable at a point $p_{0}, 0<p_{0}<$ 1. Choosing $p_{0}<a<1$, we see that $f$ is not differentiable at the point 
$a-p_{0}$. This is impossible because the set of points of the form $a-p_{0}$ forms an interval, but, according to a theorem of Lebesgue, $f$ is differentiable almost everywhere and this proves our Theorem.

With the aid of Theorem 1 we can prove the following:

Theorem 2. Let $f:(0,1) \rightarrow(0, \infty)$ and let $f$ be monotonic. Then $f$ satisfies the inequality (4) for a fixed integer $n \geqq 3$ if and only if $f$ has the form $f(p)=A p^{c}$, where $A>0$ and either $c \leqq-1$ or $c \geqq 0$.

Proof. From (16) it follows that

$$
\frac{p}{f(p)} f^{\prime}(p)=\frac{\frac{1}{2}}{f\left(\frac{1}{2}\right)} f^{\prime}\left(\frac{1}{2}\right)
$$

for all $p$ such that $0<p<1$. Indeed, if $p<\frac{1}{2}$, then the substitution $a=\frac{1}{2}+p$ gives (17) for $p<\frac{1}{2}$. If $p \geqq \frac{1}{2}$, then $a-p<\frac{1}{2}$ for $p<a<1$ and (16) gives (17) also for $p \geqq \frac{1}{2}$ since it is true for $a-p<\frac{1}{2}$. Therefore

$$
\frac{p}{f(p)} f^{\prime}(p) \equiv c
$$

for $0<p<1$.

The relation (18) shows that $f$ has to have the form

$$
f(p)=A p^{c}
$$

where $A>0$ and $c$ is constant.

Conversely, we shall show that $f(p)=A p^{c}$ where $A>0$, satisfies (4) if either $c \leqq-1$ or $c \geqq 0$, and if $-1<c<0$, then the function $f(p)=A p^{c}$ does not satisfy (4).

Indeed, if either $c=0$ or $c=-1$, then $f(p)=A p^{c}$ satisfies (4). Moreover, let either $-1>c$ or $0<c$. Then by Hölder's inequality we have

$$
\sum_{i=1}^{n} p_{i}^{1+c} q_{i}^{-c} \geqq\left[\sum_{i=1}^{n}\left(p_{i}^{1+c}\right)^{1 /(1+c)}\right]^{1+c} \cdot\left[\sum_{i=1}^{n}\left(q_{i}^{-c}\right)^{-1 / c}\right]^{-c}=1
$$

In the case $-1<c<0$, by Hölder's inequality

$$
\sum_{i=1}^{n} p_{i}^{1+c} q_{i}^{-c} \leqq 1
$$

with equality holding if and only if $p_{i}=q_{i}$ for $i=1,2, \cdots, n$, which proves our Theorem. 
4. The internal functions were introduced by Á. Császár [1], [2].

A real function $f$ defined on a interval $I=(a, b)$ is an internal function on $I$ if for all $x, y \in I$

$$
\min (f(x), f(y)) \leqq f\left(\frac{x+y}{2}\right) \leqq \max (f(x), f(y))
$$

with equality iff $f(x)=f(y)$.

It is evident from (22) that every strictly monotonic function on $I$ is internal on $I$. The converse is not true, because any solution of the equation

$$
f(x+y)=f(x)+f(y)
$$

is internal on every interval $I$, and Hamel showed the existence of nonmonotonic solutions of (23).

In this note we shall use the following three propositions of Á. Császár.

PROPOSITION 1. If $f$ is an internal function on $I=(a, b)$, then $f / R_{I}(\alpha, \beta)$ (the restriction of $f$ to the set $R_{I}(\alpha, \beta)$ ) is either strictly increasing or strictly decreasing or constant, where

$$
R_{I}(\alpha, \beta)=\{\alpha+r(\beta-\alpha): r \text { is a rational number }\} \cap I
$$

and $a<\alpha<\beta<b . \quad$ (Lemma 1 of [1].)

PRoposition 2. If $f$ is internal on the interval $(a, b)$ and if $f$ is constant, resp. strictly increasing, resp. strictly decreasing on a subinterval $J$ of $(a, b)$, then $f$ has the same property on the whole interval $(a, b)$. (Lemma 2 of [1].)

Proposition 3. If $f$ is a nonmonotonic internal function on $I=$ $(a, b)$, then the sets $\{x: f(x)>f(\alpha)$ and $x \in I\}$ and $\{x: f(x)<f(\alpha)$ and $x \in I\}$ are dense in I for any $\alpha$ such that $a<\alpha<b$. (Lemma 4 of [1].)

First we shall prove the following theorem:

THEOREM 3. If $f:(0,1) \rightarrow(0, \infty)$ satisfies the inequality (4) for a fixed $n \geqq 3$, then $f$ is internal on $\left(0, \frac{1}{2}\right]$.

Proof. Assume that $f:(0,1) \rightarrow(0, \infty)$ satisfies the inequality (4) for a fixed $n \geqq 3$. Let $0<r_{1}<r_{2} \leqq \frac{1}{2}$ and let $r_{1}+r_{2}=a$. According to Lemma $1 f$ satisfies the inequality (13) for this fixed $a$. By Lemma 2 we 
see that (13) implies (14). Putting $r_{1}=p, r_{2}=a-p$ into (14) we obtain that

$$
\min \left[f\left(r_{1}\right), f\left(r_{2}\right)\right] \leqq f\left(\frac{r_{1}+r_{2}}{2}\right) \leqq \max \left[f\left(r_{1}\right), f\left(r_{2}\right)\right]
$$

where equality holds iff $f\left(r_{1}\right)=f\left(r_{2}\right)$, which proves our Theorem.

Now, we shall prove the main result of this paper.

THEOREM 4. If $f:(0,1) \rightarrow(0, \infty)$ satisfies the inequality (4) for a fixed integer $n \geqq 3$, then $f$ is monotonic on $(0,1)$.

Proof. First we shall show that it is enough to prove that $f$ is monotonic on $\left(0, \frac{1}{2}\right)$. Indeed, let $a$ be an arbitrary number such that $\frac{1}{2} \leqq a<1$ and let $a / 2 \leqq p<a$ and $a / 2 \leqq q<a$. It follows from Lemma 1 that $f$ satisfies the inequality (13) for this fixed $a$. It can be proved from (13) that $f(a-q) \geqq f(a-p)$ implies $f(p) \geqq f(q)$. So, if $f$ is monotonic on $(0, a / 2]$, it is monotonic on $(0, a)$. Since $f$ is monotonic on $(0, a)$ for all a such that $\frac{1}{2} \leqq a<1$, we have that $f$ is monotonic on $(0,1)$.

Let us assume that $f$ is nonmonotonic on $\left(0, \frac{1}{2}\right)$. According to Theorem $3 f$ is a nonmonotonic internal function on $\left(0, \frac{1}{2}\right)$. Let us fix $a$ in such a way that $0<a<\frac{1}{2}$. According to Proposition $2 f$ is nonmonotonic on $(0, a)$. As we showed earlier, $f$ satisfies the inequality

$$
p \frac{f(p)}{f(q)}+(a-p) \frac{f(a-p)}{f(a-q)} \geqq a
$$

for all $0<p<a$ and for all $0<q<a$.

We shall show that it is possible to find $p_{\alpha}$ and $p_{\beta}$ such that $0<p_{\alpha}<a / 2,0<p_{\beta}<a / 2$ and $f / R_{(0, a)}\left(p_{\alpha}, a-p_{\alpha}\right)$ is strictly increasing and $f / R_{(0, a)}\left(p_{\beta}, a-p_{\beta}\right)$ is strictly decreasing.

Indeed, since $p+\frac{1}{2}(a-2 p)=a / 2$, we deduce that $a / 2 \in \bigcap_{0<p<a / 2} R_{(0, a)}(p, a-p)$. Therefore, if the statement were not true, then according to Proposition 1 we would have either $f(p) \leqq f(a / 2)$ for all $0<p<a / 2$ in the case when there is no $p \in(0, a / 2)$ such that $f / R_{(0, a)}(p, a-p)$ is strictly decreasing or $f(p) \geqq f(a / 2)$ for all $(a / 2)<p<$ $a$ in the case when there is no $p \in(0, a / 2)$ such that $f / R_{(0, a)}(p, a-p)$ is strictly increasing. In either case the set $\{x: f(x)>f(a / 2)$ and $x \in$ $(0, a)\}$ is not dense in $(0, a)$ which is a contradiction according to Proposition 3. In the following we fix a $p_{\alpha}$ and a $p_{\beta}$ with these properties.

If $q \in R_{(0, a)}(p, a-p)$ for some $p$ such that $0<p<a / 2$, then $q=p+r(a-2 p)$ where $r$ is a rational number. Consequently, $a-$ $q=p+(1-r)(a-2 p)$, which implies that $a-q \in R_{(0, a)}(p, a-p)$, too. 
We distinguish two cases:

(i) $\lim _{p \rightarrow+0} p f(p)=0$,

(ii) $\lim \sup _{p \rightarrow+0} p f(p)>0$.

In the case (i) let us fix $q_{0}$ in such a fashion that $0<q_{0}<a / 2$ and $q_{0} \in R_{(0, a)}\left(p_{\alpha}, a-p_{\alpha}\right)$. Since $a / 2 \in R_{(0, a)}\left(p_{\alpha}, a-p_{\alpha}\right)$ we have $f(a / 2)<$ $f\left(a-q_{0}\right)$. Let us assume, furthermore, that $0<p<a / 2$ and $p \in R_{(0, a)}\left(p_{\beta}, a-p_{\beta}\right)$. Similarly, we can deduce that $f(a / 2)>f(a-p)$, which yields

$$
\lim _{\substack{p \rightarrow+0 \\ p \in R_{(0, a)}\left(p_{\beta}, a-p_{\beta}\right)}} \frac{f(a-p)}{f\left(a-q_{0}\right)}<1-\epsilon
$$

for some $\epsilon>0$. Therefore

$$
p \frac{f(p)}{f\left(q_{0}\right)}+(a-p) \frac{f(a-p)}{f\left(a-q_{0}\right)}<a
$$

if $p \in R_{(0, a)}\left(p_{\beta}, a-p_{\beta}\right)$ and if $p$ is small enough, which is a contradiction to $(25)$.

In the case (ii) we choose $p$ in such a way that

Therefore

$$
p \in R_{(0, a)}\left(p_{\alpha}, a-p_{\alpha}\right) \text {. }
$$

$$
\lim _{\substack{p \rightarrow+0 \\ p \in R_{(0, a)\left(p_{\alpha}, a-p_{\alpha}\right)}}} p f(p)=0
$$

Combining the facts that $f$ is internal on $\left(0, \frac{1}{2}\right)$ and $f / R_{(0, a)}\left(p_{\alpha}, a-p_{\alpha}\right)$ is strictly increasing we deduce that $f / R_{\left(0, \frac{1}{2}\right)}\left(p_{\alpha}, a-p_{\alpha}\right)$ is strictly increasing. Since $R_{\left(0, \frac{1}{2}\right)}\left(p_{\alpha}, a-p_{\alpha}\right)$ is dense in $\left(0, \frac{1}{2}\right)$, there exists a $p^{\prime}$ such that $p^{\prime} \in R_{\left(0, \frac{1}{2}\right)}\left(p_{\alpha}, a-p_{\alpha}\right)-R_{(0, a)}\left(p_{\alpha}, a-p_{\alpha}\right)$, which implies that

$$
\lim _{\substack{p \rightarrow a-0 \\ p \in R_{(0, a)}\left(p_{\alpha}, a-p_{\alpha}\right)}} f(p) \leqq f\left(p^{\prime}\right)<+\infty .
$$

Since $\sup _{x \in(0, a)}\{f(x)\}=+\infty$, there exists a $q_{0} \in(0, a)$ such that $f\left(a-q_{0}\right)>2 f\left(p^{\prime}\right)$. Therefore

$$
p \frac{f(p)}{f\left(q_{0}\right)}+(a-p) \frac{f(a-p)}{f\left(a-q_{0}\right)}<a
$$

if $p \in R_{(0, a)}\left(p_{\alpha}, a-p_{\alpha}\right)$ and if $p$ is small enough, which is a contradiction to (25). Thus we have shown that if $f$ is non-monotonic on $\left(0, \frac{1}{2}\right)$, then $f$ does not satisfy (4) for a fixed integer $n \geqq 3$.

Combining our previous results we have shown the following. 
THEOREM 5. Let $f:(0,1) \rightarrow(0, \infty)$. Then $f$ satisfies the inequality (4) for a fixed positive integer $n \geqq 3$ if and only if $f$ has the form $f(p)=A p^{c}$ where $A$ is an arbitrary positive number and either $c \leqq-1$ or $c \geqq 0$.

5. In this chapter we remove the condition that $f$ is positive.

It is evident that $f:(0,1) \rightarrow(-\infty, 0)$ satisfies the inequality (4) for a fixed integer $n \geqq 2$ iff $-f$ satisfies (4) for this fixed integer.

Next we shall show that if $f:(0,1) \rightarrow R-\{0\}$ and if $f$ changes its sign, then $f$ fails to satisfy (4) for any fixed $n \geqq 2$.

Assume first that $n=2$. If there exists a $p_{0}$ such that $0<p_{0}<1$, $f\left(p_{0}\right)>0$ and $f\left(1-p_{0}\right)<0$, then

$$
p_{0} \frac{f\left(p_{0}\right)}{f\left(1-p_{0}\right)}+\left(1-p_{0}\right) \frac{f\left(1-p_{0}\right)}{f\left(p_{0}\right)}<0,
$$

i.e. $f$ does not satisfy (4). Therefore, the only case to be considered is when $\operatorname{sign} f(p)=\operatorname{sign} f(1-p)$ for every $0<p<1$. Then

$$
p_{0} \frac{f\left(p_{0}\right)}{f\left(q_{0}\right)}+\left(1-p_{0}\right) \frac{f\left(1-p_{0}\right)}{f\left(1-q_{0}\right)}<0
$$

provided that sign $f\left(p_{0}\right)+\operatorname{sign} f\left(q_{0}\right)=0$, i.e. $f$ fails to satisfy (4).

Assume now that $n \geqq 3$ and $n$ is fixed. If $p_{1}$ and $p_{2}$ exist so that $0<p_{1}<1,0<p_{2}<1, p_{1}+p_{2}<1$ and $\operatorname{sign} f\left(p_{1}\right)+\operatorname{sign} f\left(p_{2}\right)=0$, then choosing

$$
q_{1}=p_{2}, \quad q_{2}=p_{1}, \quad q_{3}=p_{3}=\cdots=q_{n}=p_{n}=\frac{1-p_{1}-p_{2}}{n-2},
$$

we have

$$
p_{1} \frac{f\left(p_{1}\right)}{f\left(p_{2}\right)}+p_{2} \frac{f\left(p_{2}\right)}{f\left(p_{1}\right)}+\left(1-p_{1}-p_{2}\right)<1
$$

i.e. $f$ does not satisfy (4). Therefore, $f$ can satisfy the inequality (4) if for all $p_{1}$ and $p_{2}$ such that $0<p_{1}<1,0<p_{2}<1, p_{1}+p_{2}<1$ we have that sign $f\left(p_{1}\right)+\operatorname{sign} f\left(p_{2}\right) \neq 0$, which implies that $f$ does not change its sign.

Thus we obtain the general solution of (4) for a fixed $n \geqq 3$.

THEOREM 6. Let $f:(0,1) \rightarrow R-\{0\}$. Then $f$ satisfies the inequal ity (4) for a fixed positive integer $n \geqq 3$ if and only if $f$ has the form $f(p)=A p^{c}$ where $A$ is an arbitrary nonzero constant and either $c \leqq-1$ or $c \geqq 0$. 


\section{REFERENCES}

1. Á. Császár, Sur une classe des fonctions non mesurables, Fund. Math., 36 (1949), 72-76.

2. - Sur les fonctions internes, non monotones, Acta Sci. Math., (Szeged) 13 (1949), 48-50.

3. P. Fischer, On the inequality $\Sigma p_{f} f\left(p_{i}\right) \geqq \Sigma p_{i} f\left(q_{i}\right)$, Metrika, 18 (1972), 199-208.

4. - On the inequality $\Sigma p_{i}\left(f\left(p_{i}\right) / f\left(q_{i}\right)\right) \leqq 1$, To appear in Canadian Math. Bulletin.

5. - On the inéquality $\Sigma g\left(p_{i}\right) f\left(p_{i}\right) \geqq \Sigma g\left(p_{i}\right) f\left(q_{i}\right)$, Aequationes Math., 10 (1974), 23-33.

6. - Sur l'inégalité $\Sigma\left[p_{i} f\left(p_{i}\right)+q_{i} f\left(q_{i}\right)\right] \geqq \Sigma\left[p_{f} f\left(q_{i}\right)+q_{i} f\left(p_{i}\right)\right]$, To appear in Periodica Mathematica Hungarica.

7. - On some new generalizations of Shannon's inequality, In preparation.

8. A. Rényi, Unpublished.

9. - On measures of entropy and informations, Proc. Fourth Berkeley Symposium on Math. Stat. Probab. 1960, Vol. I, Univ. California Press, Berkeley-Los Angeles, 547-561 (1961).

Received June 10, 1974. The author thanks the referee for his advice.

UNIVERSITY OF GUELPH 
D. E. Bennett, Strongly unicoherent continua ............................. 1

Walter R. Bloom, Sets of p-spectral synthesis ................................ 7

R. T. Bumby and D. E. Dobbs, Amitsur cohomology of quadratic extensions: Formulas and number-theoretic examples ................. 21

W. W. Comfort, Compactness-like properties for generalized weak topological sums

D. R. Dunninger and J. Locker, Monotone operators and nonlinear biharmonic boundary value problems ...

T. S. Erickson, W. S. Martindale, 3rd and J. M. Osborn, Prime nonassociative algebras

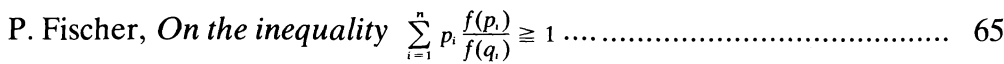

G. Fox and P. Morales, Compact subsets of a Tychonoff set ............... 75

R. Gilmer and J. F. Hoffmann, A characterization of Prüfer domains in terms of polynomials ......................................................... 81

L. C. Glaser, On tame Cantor sets in spheres having the same projection in each direction ......................................................... 87

Z. Goseki, On semigroups in which $X=X Y X=X Z X$ if and only if

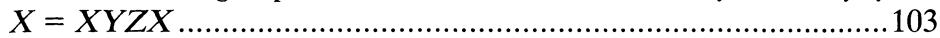

E. Grosswald, Rational valued series of exponentials and divisor

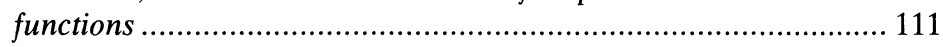

D. Handelman, Strongly semiprime rings ...................................... 115

J. N. Henry and D. C. Taylor, The $\bar{\beta}$ topology for $w^{*}$-algebras ............. 123

M. J. Hodel, Enumeration of weighted p-line arrays .......................... 141

S. K. Jain and S. Singh, Rings with quasiprojective left ideals .............. 169

S. Jeyaratnam, The diophantine equation $Y(Y+m)(Y+2 m) \times$

$$
(Y+3 m)=2 X(X+m)(X+2 m)(X+3 m) \ldots \ldots \ldots \ldots \ldots \ldots \ldots . . .183
$$

$\mathrm{R}$. Kane, On loop spaces without $p$ torsion .........................................189

Alvin J. Kay, Nonlinear integral equations and product integrals ..........203

A. S. Kechris, Countable ordinals and the analytic hierarchy, I ...........223

Ka-Sing Lau, A representation theorem for isometries of $C(X, E) \ldots \ldots . .229$

I. Madsen, On the action of the Dyer-Lashof algebra in $H_{*}(G)$..........235

R. C. Metzler, Positive linear functions, integration, and Choquet's theorem ........................................................................................ 277

A. Nobile, Some properties of the Nash blowing-up ............................297

G. E. Petersen and G. V. Welland, Plessner's theorem for Riesz conjugates 


\section{Pacific Journal of Mathematics}

\section{Vol. 60, No. $1 \quad$ September, 1975}

Donald Earl Bennett, Strongly unicoherent continua ................ 1

Walter Russell Bloom, Sets of p-spectral synthesis ................ 7

Richard Thomas Bumby and David Earl Dobbs, Amitsur cohomology of

quadratic extensions: formulas and number-theoretic examples .......

W. Wistar (William) Comfort, Compactness-like properties for generalized

weak topological sums ...................................

Dennis Robert Dunninger and John Stewart Locker, Monotone operators

and nonlinear biharmonic boundary value problems ..............

Theodore Erickson, Wallace Smith Martindale, III and J. Marshall Osborn,

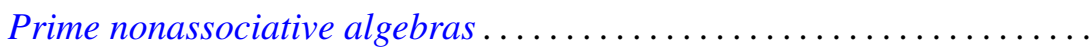

Pál Fischer, On the inequality $\sum_{i=0}^{n}\left[f\left(p_{i}\right) / f\left(q_{i}\right)\right] p_{i} \geq i \ldots \ldots \ldots \ldots \ldots$

Geoffrey Fox and Pedro Morales, Compact subsets of a Tychonoff set.......

Robert William Gilmer, Jr. and Joseph F. Hoffmann, A characterization of

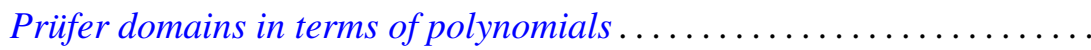

Leslie C. Glaser, On tame Cantor sets in spheres having the same projection

in each direction . ...................................

Zensiro Goseki, On semigroups in which $x=x y x=x z x$ if and only if

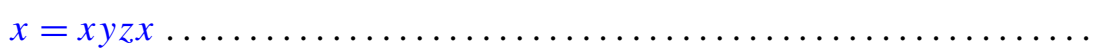

Emil Grosswald, Rational valued series of exponentials and divisor

functions.

David E. Handelman, Strongly semiprime rings

Jackson Neal Henry and Donald Curtis Taylor, The $\bar{\beta}$ topology for

$W^{*}$-algebras

Margaret Jones Hodel, Enumeration of weighted p-line arrays ...

Surender Kumar Jain and Surjeet Singh, Rings with quasi-projective left

ideals.

S. Jeyaratnam, The Diophantine equation

$$
Y(Y+m)(Y+2 m)(Y+3 m)=2 X(X+m)(X+2 m)(X+3 m) \ldots
$$

Richard Michael Kane, On loop spaces without $p$ torsion

Alvin John Kay, Nonlinear integral equations and product integrals ...

Alexander S. Kechris, Countable ordinals and the analytical hierarchy.

$I$.

Ka-Sing Lau, A representation theorem for isometries of $C(X, E)$

Ib Henning Madsen, On the action of the Dyer-Lashof algebra in $H_{*}(G)$

Richard C. Metzler, Positive linear functions, integration, and Choquet's

theorem.

Augusto Nobile, Some properties of the Nash blowing-up

Gerald E. Peterson and Grant Welland, Plessner's theorem for Riesz. 